\title{
Estimação da localização de um robô não holonômico utilizando modelo oculto de Markov (HMM) e o filtro de Kalman estendido (EKF).
}

\author{
Neemias Silva Monteiro* Carlos Andrey Maia* \\ Vinicius Mariano Gonçalves* \\ * Programa de Pós-Graduação em Engenharia Elétrica - Universidade \\ Federal de Minas Gerais - Av. Antônio Carlos 6627, 31270-901, \\ Belo Horizonte, MG, Brasil(e-mails: neemias@ufmg.br, \\ \{maia,mariano\}@cpdee.ufmg.br).
}

\begin{abstract}
Knowing the location of a robot is fundamental to correctly planning a safe navigation towards the destination. In this article we use the hidden Markov model (HMM) and the extended Kalman filter (EKF) to estimate the position of a non-holonomic mobile robot, during the tracing of parametrized trajectories in time. The proposed location system consists of a transceiver coupled to the robot, which communicates with three other transceivers in predetermined positions. By sending periodic messages to other transceivers, the robot can estimate its position relative to other transceivers. These measurements are used in HMM and EKF to estimate the location of the robot. Experiments were performed, and the results show that the methods used present robustness to estimate the location of robots.

Resumo: Conhecer a localização de um robô é fundamental para planejar corretamente uma navegação segura em direção ao local de destino. Neste artigo utiliza-se o modelo oculto de Markov (HMM) e o filtro de Kalman estendido (EKF) para estimar a posição de um robô móvel não holonômico, durante o rastreamento de trajetórias parametrizadas no tempo. O sistema de localização proposto é constituído de um transceptor acoplado ao robô, que se comunica com outros três transceptores em posições predeterminadas. Ao enviar mensagens periódicas aos outros transceptores, o robô consegue estimar sua posição relativa aos demais transceptores. Estas medidas são usadas no HMM e no EKF para estimar a localização do robô. Experimentos foram realizados, e os resultados mostram que os métodos utilizados apresentam robustez para estimar a localização de robôs.
\end{abstract}

Keywords: Extended Kalman Filter; Hidden Markov Model; Localization; Robotics.

Palavras-chaves: Filtro de Kalman Estendido; Modelo Oculto de Markov; Localização; Robótica.

\section{INTRODUÇÃO}

Com o avançar dos anos, é notável o aumento da demanda por sistemas robóticos. Os robôs estão cada vez mais presentes em nosso cotidiano, e podem realizar tarefas com rapidez e precisão. A tarefa mais simples atribuída a um robô móvel é sair de um local inicial, e ir em direção a um local de destino. Entretanto, o ambiente por onde o robô se locomove é imprevisível e dinâmico, implicando em altas incertezas durante a navegação. Para planejar corretamente a navegação de um robô em direção ao seu destino, se faz necessário saber onde o robô está localizado no mapa do ambiente. Se a posição do robô no ambiente não é conhecida, ele não pode realizar nenhuma tarefa de navegação. Em muitas aplicações, utiliza-se odometria para estimar a localização do robô no ambiente. Porém,

\footnotetext{
* Os autores agradecem as agências CNPq, CAPES, FAPEMIG, e ao Programa de Pós-Graduação em Engenharia Elétrica da UFMG, pelo apoio financeiro.
}

se o robô operar por longos períodos, a estimativa de posição (fornecida pela odometria) não será precisa devido ao acúmulo de erros (Chong and Kleeman, 1997). Logo, deve-se estabelecer um método que seja responsável por estimar a localização do robô durante seu trajeto.

O presente trabalho utiliza duas estruturas probabilísticas para realizar o rastreamento da posição de um robô móvel planar não holonômico: o HMM (hidden Markov model) e o EKF (extended Kalman filter). Na literatura encontram-se várias pesquisas utilizando o HMM e o EKF para localizar robôs a partir de leituras de sensores (Tong et al., 2018; Savage et al., 2018; Pomárico-Franquiz et al., 2014; Lee et al., 2011). Experimentos são realizados no software VREP, no qual são passadas algumas trajetórias a serem seguidas pelo robô, e compara-se o resultado da estimação da posição obtido com as duas estruturas. O robô é equipado com um transceptor, que se comunica com outros três transceptores fixados em posições predeterminadas do ambiente de trabalho. Cada vez que o transceptor do 
robô envia uma mensagem aos demais, afere-se a distância relativa do robô em relação aos transceptores. Estas três distâncias são utilizadas como observação indireta do estado do robô no HMM e como medição (após realizada a trilateração) na fase de atualização do EKF.

O artigo está organizado da seguinte forma: na Seção 2 apresenta-se a estrutura do HMM e na Seção 3 a do EKF. A Seção 4 mostra uma visão geral de como o sistema foi desenvolvido. A Seção 5 exibe resultados de simulações realizadas no V-REP. Conclusões obtidas e trabalhos futuros são relatados na Seção 6.

\section{LOCALIZAÇÃO BASEADA EM MODELO OCULTO DE MARKOV (HMM)}

Um modelo de Markov é um modelo estocástico usado para modelar a evolução de um sistema não determinístico, como o ambiente em torno do robô e o estado do próprio robô (Peter, 2016). Os modelos markovianos têm a propriedade de que a distribuição de probabilidade do próximo estado $s_{t+1}$, depende apenas do estado atual $s_{t}$ e não da sequência de eventos que o precederam $s_{1: t-1}$. Desta forma, os estados anteriores são irrelevantes para predição de estados futuros, isto é, $p\left(s_{t+1} \mid s_{1: t}\right)=p\left(s_{t+1} \mid s_{t}\right)$.

Existem algumas variações do modelo de Markov que podem ser utilizadas em diferentes situações, dependendo se o estado é totalmente observável, e se robô pode afetar este estado tomando decisões. O modelo de Markov mais simples é a cadeia de Markov. Ela modela um sistema totalmente observável, por exemplo, o ambiente em torno de um robô equipado com um sensor de observação perfeito. Uma cadeia de Markov pode ser definida por uma tupla: $(S, T)$, no qual $S$ é o conjunto de estados e $T$ é a probabilidade de transição entre os estados (Peter, 2016). As probabilidades de transição podem ser representadas na forma de uma matriz, denominada matriz de transição $A$ (Cassandras and Lafortune, 2009):

$$
A \equiv\left[a_{i j}\right] \quad i, j=1,2, \ldots, N
$$

em que, $a_{i j} \in[0,1]$ representa a probabilidade de transição do estado $s_{i}$ para o estado $s_{j}$, e $\sum_{j} a_{i j}=1 \forall$ estado $s_{i}$.

\subsection{Modelo Oculto de Markov (HMM)}

Ao modelar sistemas complexos do mundo real o conjunto $S$ raramente é observável, isso ocorre devido à oclusões naturais ou observações imperfeitas do sensor (Peter, 2016). Tais situações podem ser modeladas por um modelo oculto de Markov (HMM). O HMM é um modelo de Markov no qual os estados não são diretamente observáveis, ou seja, ocultos. Na cadeia de Markov considera-se que cada estado corresponde a uma observação, já o HMM representa o cenário em que a observação é uma distribuição de probabilidade dos estados (Rabiner, 1990). O HMM é modelado na Figura 1. Os estados $S$ são ocultos, e as únicas informações fornecidas são as observações do sensor $z_{t}$. Assim, uma resolução com HMM pode se resumir a determinar $p\left(s_{t} \mid z_{t}\right)$. Um exemplo de HMM aplicado em robótica é: determinar a localização de um robô em um ambiente, sendo que as observações do espaço de trabalho são provenientes do sistema de percepção do robô. Nesta aplicação, os estados seriam "observados indiretamente" por meio das informações do sensor $z_{t}$.

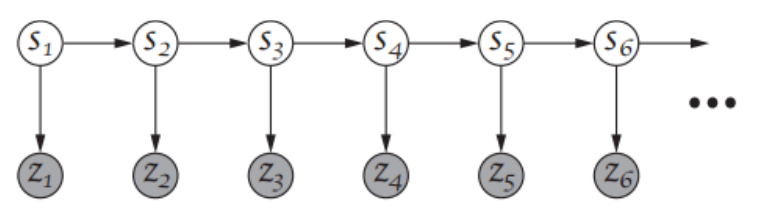

Figura 1. Modelo gráfico para o HMM (Peter, 2016).

O HMM é representado pela notação $\lambda=\{A, B, \pi\}$. A matriz, $A$, representa a distribuição de probabilidade de transição de estado, $B$, representa a distribuição de probabilidade de observação e, $\pi$, a distribuição de probabilidade do estado inicial. Com a formulação do HMM, exitem três problemas que devem ser solucionados para que este tipo de modelo seja utilizado no mundo real (Rabiner, 1990):

Problema 1. Dada a sequência de observações $O=$ $o_{1}, o_{2}, \ldots, o_{t}$, e um modelo $\lambda$, como calcular de forma eficiente $p(O \mid \lambda)$ ?

Problema 2. Dada a sequência de observações $O=$ $o_{1}, o_{2}, \ldots, o_{t}$, e um modelo $\lambda$, como escolher a sequência de estados correspondente $S=s_{1}, s_{2}, \ldots, s_{t}$ que é ótima em algum sentido?

Problema 3. Como os parâmetros do modelo $\lambda=\{A, B, \pi\}$ podem ser ajustados para maximizar $p(O \mid \lambda)$ ?

O Problema 1 resume-se a calcular a probabilidade de que a sequência observada tenha sido produzida pelo modelo $\lambda$, e é frequentemente resolvido por meio do procedimento forward-backward. No Problema 3, tenta-se otimizar os parâmetros do modelo, de modo a descrever melhor como uma dada sequência de observações ocorre. A resolução dos Problemas 1 e 3 não se encontra no escopo do presente trabalho. No Problema 2, deseja-se descobrir a parte oculta do modelo, ou seja, encontrar a sequência "correta" de estados percorrida pelo robô durante o trajeto. Assim, a resolução deste problema é aconselhável para o rastreamento dos estados, uma vez que fornece um histórico de estados mais prováveis, dada uma sequência de observações. Existem várias formas de resolver o Problema 2, pois há vários critérios de otimalidade que podem ser escolhidos. Um método de encontrar a melhor solução é o algoritmo de Viterbi (Forney, 1973; Viterbi, 1967).

\subsection{Algoritmo de Viterbi}

Para encontrar a melhor sequência de estados (única) $S=s_{1} s_{2} \ldots s_{t}$, para uma dada sequência de observações $O=o_{1} o_{2} \ldots o_{t}$, necessita-se definir a grandeza:

$$
\delta_{t}(i)=\max _{s_{1}, \ldots, s_{t-1}} p\left[s_{1} \ldots s_{t}=i, O=o_{1} \ldots o_{t} \mid \lambda\right],
$$

isto é, $\delta_{t}(i)$ representa a probabilidade da melhor sequência de estados, que leva ao estado $s_{i}$ no instante $t$, com as $t$ primeiras observações. Por indução temos:

$$
\delta_{t+1}(j)=\left[\max _{i} \delta_{t}(i) a_{i j}\right] b_{j}\left(o_{t+1}\right)
$$

em que, $b_{j}\left(o_{t+1}\right)$ é a probabilidade de se observar $o_{t+1}$ no estado $s_{j}$.

Para obter a sequência de estados, é necessário manter o rastreamento do argumento que maximizou (3), para cada $t$ e $j$. O procedimento para encontrar a melhor sequência de estados pode ser dividido em quatro etapas, como mostra o Algoritmo 1 (Rabiner, 1990): 


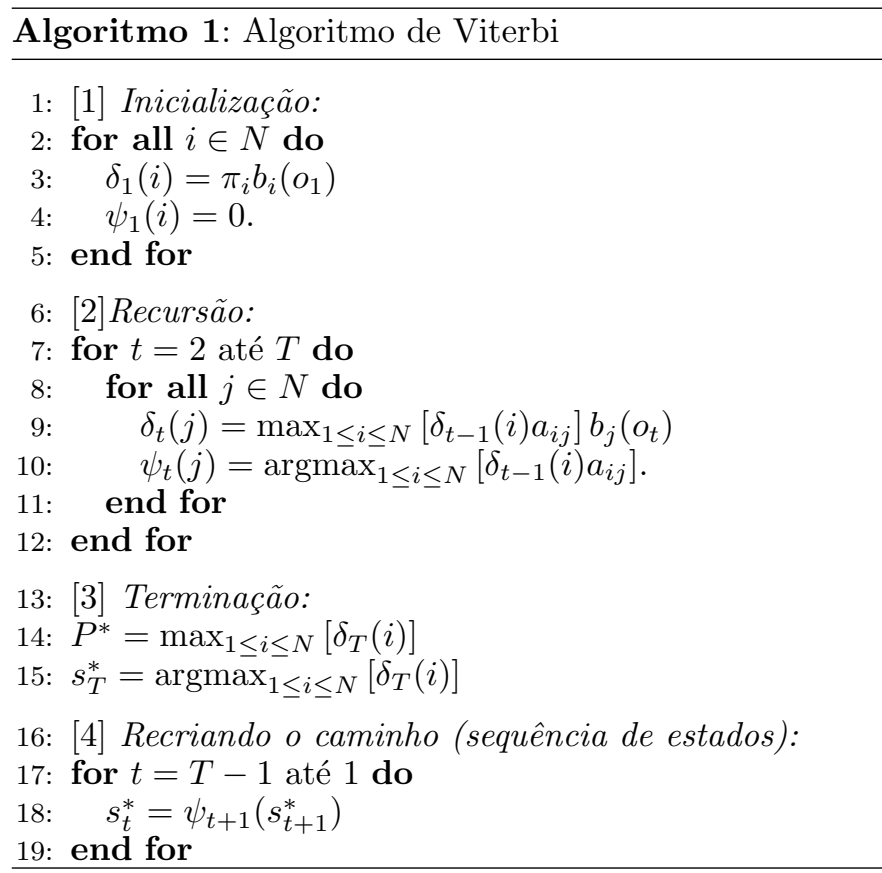

\section{LOCALIZAÇÃO BASEADA EM FILTRO DE KALMAN ESTENDIDO (EKF)}

O filtro de Kalman (KF) é um filtro recursivo que estima o estado de um sistema dinâmico linear, a partir de uma série de medições ruidosas. Para gerar uma estimativa dos estados, o KF utiliza o modelo do sistema, as entradas de controle conhecidas e as medições. Em suma, o KF combina uma predição do estado de um sistema com uma nova medida usando média ponderada (atualização). O resultado da média é uma nova estimativa do estado, que se localiza entre o estado predito e o medido, apresentando uma melhor incerteza estimada que qualquer um dos dois unicamente. O processo é repetido a cada iteração, com a nova estimativa e sua covariância gerando a predição usada na iteração seguinte (Thrun et al., 2005).

O filtro de Kalman estendido (EKF) é usado quando o sistema apresenta não linearidade. O EKF lineariza o modelo em torno da estimação atual e em seguida utiliza as equações do KF tradicional. O EKF é baseado na linearização da função de transição de estados e da função de medição por meio da aproximação por série de Taylor, fazendo o trucamento no termo de primeira ordem, desprezando os termos de ordem superior. Esta aproximação pode causar problemas se as não linearidades são muito grandes, fazendo o algoritmo divergir. Desta forma, pode-se dizer que o EKF é sub-ótimo e sem garantias de convergência (Särkkä, 2013; Thrun et al., 2005). O EKF é mostrado no Algoritmo 2.

Tanto o KF, como o EFK representam a crença no tempo $t$ (no paradigma probabilístico, a crença é a estimativa momentânea da posição do robô). A crença é representada pela média $\mu_{t}$ e a covariância $\Sigma_{t}$. A entrada do EKF é a crença no tempo $t-1$, representada por $\mu_{t-1}$ e $\Sigma_{t-1}$. Para atualizar estes parâmetros utiliza-se as entradas de controle $u_{t}$ e as medições $z_{t}$. A saída do EKF é a crença no tempo $t$, representada por $\mu_{t}$ e $\Sigma_{t}$. A variável $K_{t}$ representa ganho de Kalman, e especifica o grau em que a medida $z_{t}$ é incorporada na nova estimativa do estado. Na linha 6 ,

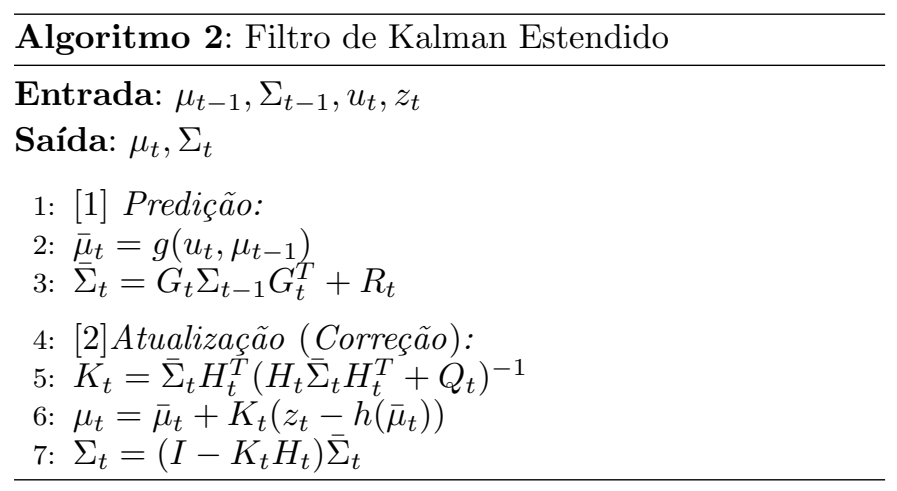

manipula-se a média $\bar{\mu}_{t}$, ajustando-a proporcionalmente ao ganho $K_{t}$ e ao desvio da atual medida $z_{t}$. Por fim, calculase na linha 7 a nova covariância da crença. Os ruídos do processo e da medição são assumidos como independentes e normalmente distribuídos com covariâncias $R_{t}$ e $Q_{t}$, respectivamente (Thrun et al., 2005).

Para o caso de um robô diferencial planar (não holonômico), em um ambiente sem obstáculos, o modelo do robô $g\left(u_{t}, \mu_{t-1}\right)$, utilizado para encontrar $\bar{\mu}_{t}$ é:

$$
\begin{gathered}
x_{t}=x_{t-1}+T v_{t} \cos \left(\theta_{t-1}\right), \\
y_{t}=y_{t-1}+T v_{t} \operatorname{sen}\left(\theta_{t-1}\right), \\
\theta_{t}=\theta_{t-1}+T \omega_{t} .
\end{gathered}
$$

O parâmetro $T$ é o tempo de amostragem, $(v, \omega)$ as velocidades linear e angular aplicadas ao robô, e $(x, y, \theta)$ é a estimação da pose do robô. Assim, em cada iteração, $\bar{\mu}_{t}=$ $\left(x_{t}, y_{t}, \theta_{t}\right)$. Pode-se observar por (4) a (6) que $g\left(u_{t}, \mu_{t-1}\right)$ é não linear, esta não linearidade distorce a crença. A linearização aproxima $g\left(u_{t}, \mu_{t-1}\right)$ por uma função linear. Quando $g\left(u_{t}, \mu_{t-1}\right)$ é linearizada as mecânicas de propagação de crenças são equivalentes às do KF. Utilizando a expansão de Taylor (de primeira ordem), constrói-se uma aproximação linear para uma função $g\left(u_{t}, \mu_{t-1}\right)$, e consequentemente determina-se a matriz $G_{t}$ do Algoritmo 2:

$$
\begin{gathered}
G_{t}=\left.\frac{\partial g\left(u_{t}, \mu_{t-1}\right)}{\partial \mu}\right|_{\mu=\mu_{t-1}}=\left[\begin{array}{lll}
\frac{\mathrm{d} x_{t}}{\mathrm{~d} x} & \frac{\mathrm{d} x_{t}}{\mathrm{~d} y} & \frac{\mathrm{d} x_{t}}{\mathrm{~d} \theta} \\
\frac{\mathrm{d} y_{t}}{\mathrm{~d} x} & \frac{\mathrm{d} y_{t}}{\mathrm{~d} y} & \frac{\mathrm{d} y_{t}}{\mathrm{~d} \theta} \\
\frac{\mathrm{d} \theta_{t}}{\mathrm{~d} x} & \frac{\mathrm{d} \theta_{t}}{\mathrm{~d} y} & \frac{\mathrm{d} \theta_{t}}{\mathrm{~d} \theta}
\end{array}\right]_{\mu=\mu_{t-1}}, \\
G_{t}=\left[\begin{array}{ccc}
1 & 0 & -T v_{t} \operatorname{sen}\left(\theta_{t-1}\right) \\
0 & 1 & T v_{t} \cos \left(\theta_{t-1}\right) \\
0 & 0 & 1
\end{array}\right]_{\mu=\mu_{t-1}} .
\end{gathered}
$$

Nota-se agora que a matriz $G_{t}$ é linear, pois $\operatorname{sen}\left(\theta_{k-1}\right)$ e $\cos \left(\theta_{k-1}\right)$ são valores numéricos. A matriz $G_{t}$ não é constante e deve ser atualizada a cada iteração. O mesmo raciocínio de linearização aplicado à $g\left(u_{t}, \mu_{t-1}\right)$ é válido para função de medição $h\left(\bar{\mu}_{t}\right)$ (e consequentemente $H_{t}$ ).

\section{VISÃO GERAL DO SISTEMA}

Neste trabalho, implementa-se no V-REP um sistema de localização para um robô móvel não holonômico, em um ambiente (sem obstáculos) de dimensão $5 \times 5 m$. O robô utilizado é o Pioneer 3-DX, ele se locomove pelo ambiente de acordo com a equação paramétrica escolhida. A estimação da posição $(x, y)$ do robô é obtida por meio da comunicação de um transceptor acoplado ao Pioneer 3-DX, com outros três transceptores fixados em posições predeterminadas. 
Um transceptor é um dispositivo que combina um transmissor e um receptor em apenas um aparelho. O transceptor acoplado ao robô envia periodicamente mensagens aos demais transceptores, e estima a distância relativa do robô para os outros três transceptores baseado no tempo de resposta destes terminais. Foi escolhido fixar três transceptores, pois este é o número mínimo de terminais que devem ser espalhados pelo ambiente de trabalho do robô para que seja possível especificar a posição do robô utilizando a estratégia de trilateração.

A Figura 2 exibe o sistema de localização proposto. A Figura 2(a) mostra a imagem do sistema antes de iniciar a simulação, e a Figura 2(b) mostra uma imagem do sistema capturada durante a simulação. Os feixes amarelos da Figura 2(b) representam o fluxo de dados entre os transceptores, e as linhas amarelas tracejadas as distâncias relativas entre o transceptor acoplado no robô aos demais.

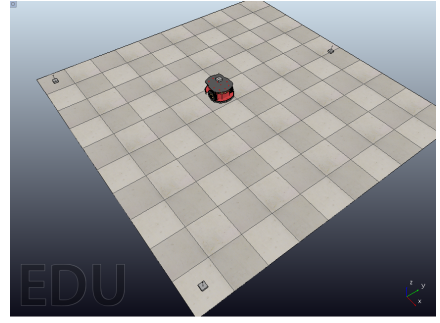

(a) Antes de iniciar a simulação.

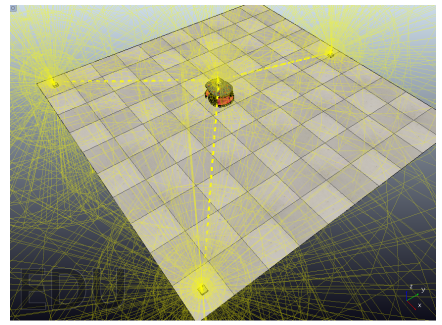

(b) Durante a simulação.
Figura 2. Sistema de localização proposto.

Para estimar em qual estado do ambiente o robô está localizado, faz-se uso do algoritmo de Viterbi (Algoritmo 1) e do EKF (Algoritmo 2). Nas próximas subseções mostra-se detalhes de como as três distâncias relativas (medidas pelo robô) são aplicadas nos algoritmos. As distâncias relativas do robô aos transceptores são denotadas por $d_{1}, d_{2}$ e $d_{3}$ e a posição dos transceptores por $\left(x_{1}, y_{1}\right),\left(x_{2}, y_{2}\right)$ e $\left(x_{3}, y_{3}\right)$. Sendo o tempo de cada iteração da simulação no V-REP de $50 \mathrm{~ms}$, e o ciclo de leitura da medição das distâncias (do robô aos demais transceptores) de 500ms.

\subsection{Observações Indiretas no HMM}

Para resolver o algoritmo de Viterbi é necessário discretizar o espaço de trabalho e obter o modelo do sistema $\lambda=\{A, B, \pi\}$. Na discretização divide-se o espaço de trabalho em células, e associa-se à cada célula a probabilidade do robô estar na localização correspondente à célula. Cada uma das células equivale a um estado do ambiente. No presente trabalho, optou-se por dividir o mapa $5 \times 5 \mathrm{~m} \mathrm{em}$ 900 estados de tamanho $16.67 \times 16.67 \mathrm{~cm}$.

A matriz de transição de estados $A$ é construída de modo que estados adjacentes recebem peso 1 , e estados que não estão diretamente conectados peso 0 . Os valores da matriz $\pi$ correspondem às probabilidades dos estados iniciais. $\mathrm{O}$ valor correspondente ao estado que o robô está inicialmente localizado recebe valor 1 (em $\pi$ ), enquanto os demais estados recebem valor 0 . A inferência do verdadeiro estado que o robô está localizado será feita de forma indireta por meio das distâncias $d_{1}, d_{2}, d_{3}$. Estas distâncias são utilizadas para determinar a probabilidade $b_{j}\left(o_{t}\right)$ presente na linha 9 do Algoritmo 1:

$$
b_{j}\left(o_{t}\right)=b_{j}\left(d_{1}, d_{2}, d_{3}\right)=p\left(s_{j} \mid d_{1}\right) p\left(s_{j} \mid d_{2}\right) p\left(s_{j} \mid d_{3}\right),
$$
sendo,

$$
\begin{gathered}
p\left(s_{j} \mid d_{l}\right)=\frac{d_{l}-\left|R_{l}-d_{l}\right|}{d_{l}}, \\
R_{l}=\sqrt{\left(s_{j x}-x_{l}\right)^{2}+\left(s_{j y}-y_{l}\right)^{2}},
\end{gathered}
$$

para $l=1,2,3$. A expressão (10) é válida para:

$$
d_{l}-\Delta \leq R_{l} \leq d_{l}+\Delta,
$$

no qual, $\Delta=0.2 \mathrm{~m}$. Caso (12) seja falsa, $p\left(s_{j} \mid d_{l}\right)=0$.

\subsection{Medições no EKF}

A fase de predição do EKF é realizada a cada ciclo da simulação, já a de atualização é realizada apenas quando há chegada de um novo conjunto de medidas. Ou seja, a atualização é realizada a cada 10 iterações da simulação. A medida $z_{t}$ (atualização) é composta pelas distâncias $d_{1}, d_{2}, d_{3}$. Estas distâncias precisam ser convertidas para um ponto $(x, y)$ no plano, para tal faz-se uso da trilateração. A trilateração é utilizada quando se conhece as posições de três referências, e as distâncias delas até o ponto que se deseja estimar, como mostra a Figura 3.

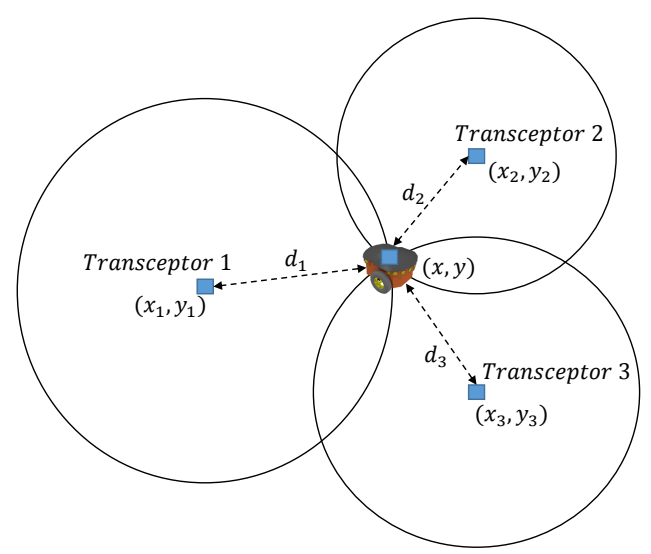

Figura 3. Trilateração utilizando transceptores.

A trilateração se dá pela intersecção dos três círculos centrados nas posições dos transceptores, tendo o raio igual a cada medição de alcance $\left(d_{1}, d_{2}, d_{3}\right)$. Para cada transceptor $i$ temos: $\left(x-x_{i}\right)^{2}+\left(y-y_{i}\right)^{2}=d_{i}^{2}$. Este sistema de equações pode ser rearranjando de modo a obter:

$$
\left\{\begin{array}{l}
\left(x^{2}+y^{2}\right)-2 x_{1} x-2 y_{1} y=d_{1}^{2}-x_{1}^{2}-y_{1}^{2} \\
\left(x^{2}+y^{2}\right)-2 x_{2} x-2 y_{2} y=d_{2}^{2}-x_{2}^{2}-y_{2}^{2} \\
\left(x^{2}+y^{2}\right)-2 x_{3} x-2 y_{3} y=d_{3}^{2}-x_{3}^{2}-y_{3}^{2}
\end{array},\right.
$$

ou em notação matricial:

$$
\left[\begin{array}{c}
1-2 x_{1}-2 x_{1} \\
1-2 x_{2}-2 y_{2} \\
1-2 x_{3}-2 y_{3}
\end{array}\right]\left[\begin{array}{c}
x^{2}+y^{2} \\
x \\
y
\end{array}\right]=\left[\begin{array}{c}
d_{1}^{2}-x_{1}^{2}-y_{1}^{2} \\
d_{2}^{2}-x_{2}^{2}-y_{2}^{2} \\
d_{3}^{2}-x_{3}^{2}-y_{3}^{2}
\end{array}\right]
$$

Assim, (14) pode ser representada por $A x=b$. A solução $\hat{x}$ pode ser determinada no sentido do método de mínimos quadrados (Norrdine, 2012):

$$
\hat{x}=\left(A^{T} A\right)^{-1} A^{T} b .
$$

Portanto, $\hat{x}$ contém a estimativa da posição do robô $(x, y)$, a partir da trilateração, e será usada para representar a medida $z_{t}$ no Algoritmo 2. 


\subsection{Trajetórias e Planejamento de Movimento}

As referências a serem seguidas pelo robô são trajetórias parametrizadas pelo tempo. Neste trabalho, estima-se a localização do robô (usando os Algoritmos 1 e 2) para as trajetórias mostradas de (16) a (18). Em (16) tem-se a trajetória de um "quadrado", em (17) é apresentada a curva do "oito", e em (18) o "caracol de Pascal".

$$
\left\{\begin{array}{cc}
x_{d}(t)=(6 / \pi) \alpha t-1.5, \quad y_{d}(t)=-1.5, \quad t \in\left[0, \frac{\pi}{2 \alpha}\right] \\
x_{d}(t)=1.5, \quad y_{d}(t)=(6 / \pi) \alpha t-4.5, \quad t \in\left[\frac{\pi}{2 \alpha}, \frac{\pi}{\alpha}\right] \\
x_{d}(t)=-(6 / \pi) \alpha t+7.5, \quad y_{d}(t)=1.5, \quad t \in\left[\frac{\pi}{\alpha}, \frac{3 \pi}{2 \alpha}\right] \\
x_{d}(t)=-1.5, \quad y_{d}(t)=-(6 / \pi) \alpha t+10.5, \quad t \in\left[\frac{3 \pi}{2 \alpha}, \frac{2 \pi}{\alpha}\right]
\end{array} .\right.
$$

$$
\begin{gathered}
\left\{\begin{array}{c}
x_{d}(t)=1.8 \cos (\alpha t) \\
y_{d}(t)=\operatorname{sen}(2 \alpha t)
\end{array} .\right. \\
\left\{\begin{array}{c}
r=2(\operatorname{sen}(\alpha t)+0.4) \\
x_{d}(t)=r \cos (\alpha t) \\
y_{d}(t)=r \operatorname{sen}(\alpha t)-1.7
\end{array} .\right.
\end{gathered}
$$

De (16) a (18) descreve-se o rastreamento da posição do robô durante a navegação, para $t \in\left[0, \frac{2 \pi}{\alpha}\right]$. O par $\left(x_{d}(t), y_{d}(t)\right)$ é a referência a ser seguida por um controlador feedback linearization ${ }^{1}$, e $\left(\dot{x}_{d}(t), \dot{y}_{d}(t)\right)$ a componente feedforward. O controlador fornece as velocidades $(v, \omega)$ que serão aplicadas ao Pioneer 3-DX:

$$
\begin{gathered}
{\left[\begin{array}{l}
\dot{x}_{r} \\
\dot{y}_{r}
\end{array}\right]=\left[\begin{array}{l}
\dot{x}_{d}(t) \\
\dot{y}_{d}(t)
\end{array}\right]+k_{p} \cdot\left[\begin{array}{c}
x_{d}(t)-x_{r} \\
y_{d}(t)-y_{r}
\end{array}\right],} \\
{\left[\begin{array}{c}
v \\
\omega
\end{array}\right]=\left[\begin{array}{cc}
\cos \left(\theta_{r}\right) & \operatorname{sen}\left(\theta_{r}\right) \\
-\operatorname{sen}\left(\theta_{r}\right) / d & \cos \left(\theta_{r}\right) / d
\end{array}\right] \cdot\left[\begin{array}{l}
\dot{x}_{r} \\
\dot{y}_{r}
\end{array}\right] .}
\end{gathered}
$$

O ganho do controlador é representado por $k_{p}$ e $\left(x_{r}, y_{r}, \theta_{r}\right)$ é a informação obtida pelo "ground truth" do V-REP. O parâmetro $d$ é um ponto próximo ao centro do robô, e é usado para evitar singularidades.

\section{RESULTADOS E DISCUSSÕES}

Nesta seção, são mostrados os resultados obtidos com experimentos no V-REP. Nos testes a seguir, considerouse $\alpha=0.2$, e que a posição inicial do robô é representada por um triângulo ciano, e a posição final por um triângulo amarelo. Vídeos ilustrativos estão disponíveis em https://www . youtube. com/playlist?list= PLk8QJI104xOCBLMeWa9Zh0surkhCmcJ8N.

Na Figura 4 é exibida a estimação da posição do robô não holonômico para trajetória do "quadrado" (usando o HMM e o EKF), juntamente com a trajetória percorrida pelo robô. Nota-se que o robô foi lançado um pouco distante da trajetória do "quadrado", e por esta razão ele leva algum tempo até convergir para mesma. No início da estimação, são coletadas poucas medições dos transceptores, e por isto o resultado da estimação do HMM e do EKF não é bom. Conforme são recebidas mais medições, o resultado dos estimadores se aproxima da trajetória real do robô. Os estimadores tornam a apresentar oscilações quando o robô executa uma curva muito acentuada, porém com aquisição de mais medições a estimação é aprimorada, e os estimadores passam a convergir para a trajetória que o robô está executando.

1 Mais informações sobre o feedback linearization são apresentadas no livro "Robot modeling and control" de Spong et al. (2006).

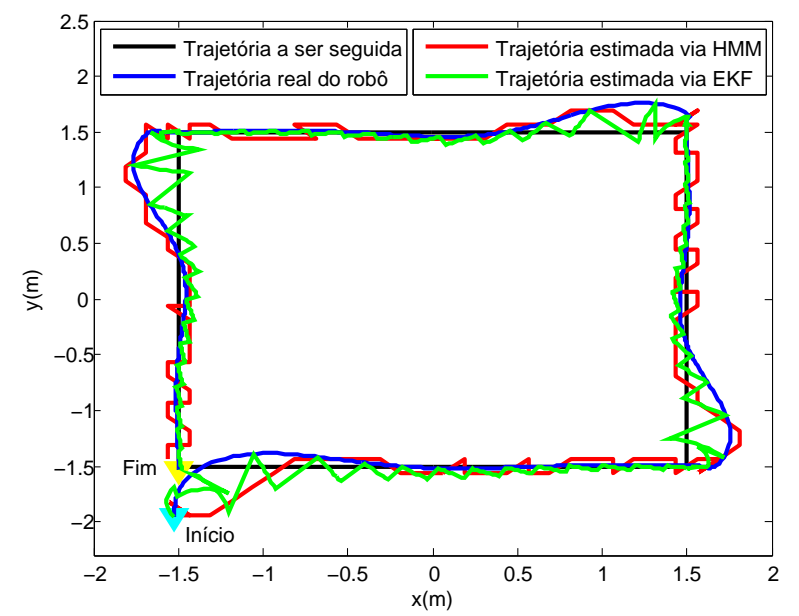

Figura 4. Localização: trajetória do "quadrado".

A Figura 5 mostra o resultado da estimação da posição do robô para trajetória do "oito". Observa-se que a estimação do EKF, após um pequeno período transitório, converge para trajetória executada pelo robô, ficando praticamente sobreposta à mesma. O HMM também apresentou bons resultados, porém com pequenas oscilações em torno da trajetória real. A Figura 6 expõe a estimação da posição do robô para trajetória do "caracol de Pascal". Os resultados foram semelhantes aos da Figura 5, porém os estimadores demoram mais tempo até convergir para a trajetória executada pelo robô.

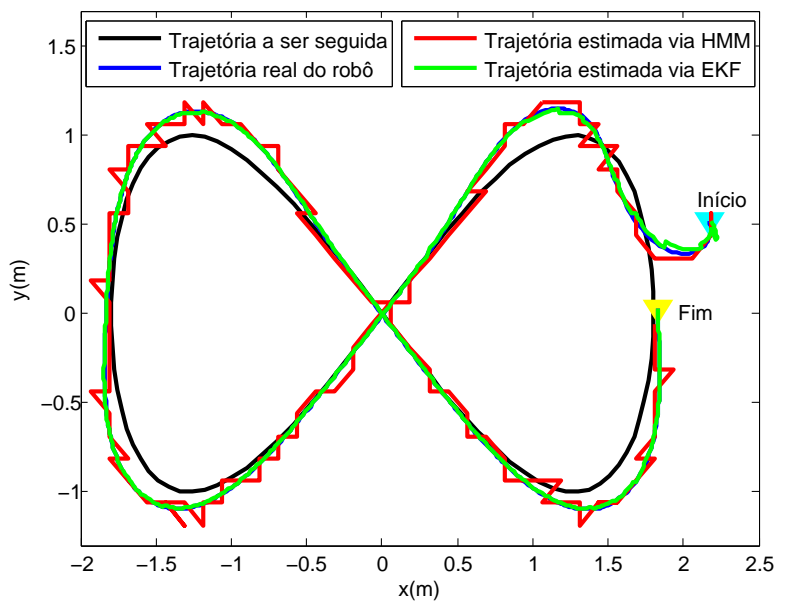

Figura 5. Localização: trajetória do "oito".

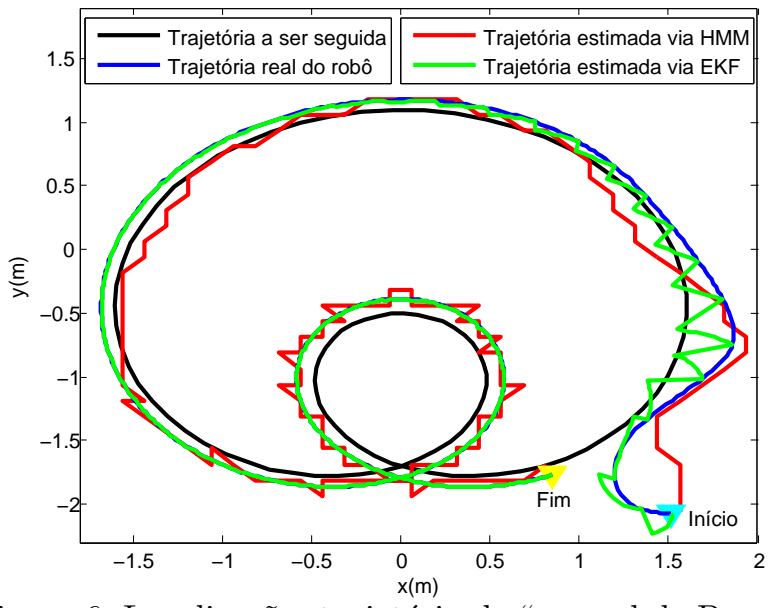

Figura 6. Localização: trajetória do "caracol de Pascal". 
Na Figura 7 mostra-se a distância entre a posição que o robô estava em determinado instante de tempo, e a posição fornecida pelos estimadores HMM e EKF. Na Figura $7(\mathrm{a})$, pode-se ver claramente a oscilação inicial apresentada pelos estimadores na trajetória do "quadrado", e os outros três momentos de curva acentuada. Pela Figura 7(a) observa-se que o HMM apresenta melhor resultado que o EKF somente nos períodos de curva acentuada. Na trajetória do "quadrado", o erro entre as distâncias estimadas e real chega a $0.5 \mathrm{~m}$. Já na Figura 7(b), notase que a amplitude do erro do estimador HMM é pequena, enquanto o erro do EKF é praticamente nulo (após os 5 segundos). O resultado da Figura 7(b) se mantém na Figura $7(\mathrm{c})$, entretanto os erros têm amplitudes maiores.

(a) Trajetória do "quadrado"

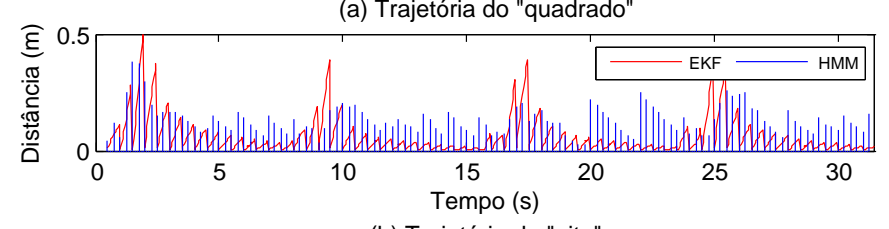

(b) Trajetória do "oito"

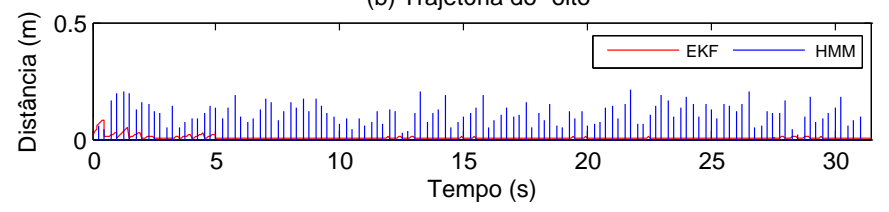

(c) Trajetória do "caracol de Pascal"

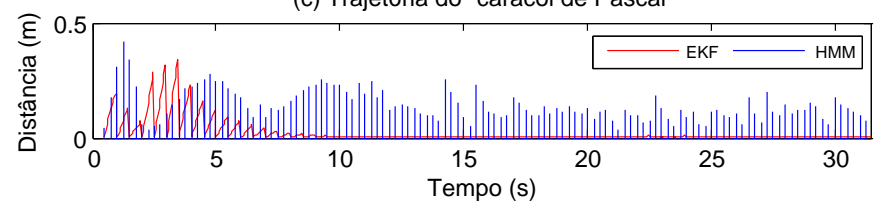

Figura 7. Distância entre a posição real do robô e a posição fornecida pelos estimadores HMM e EKF.

\section{CONCLUSÕES E TRABALHOS FUTUROS}

Neste trabalho o HMM e o EKF foram apresentados como alternativa para resolver o problema da estimação da posição de um robô planar não holonômico. A estratégia foi utilizar uma estrutura perceptiva, a partir de informações de transceptores, para obter informações relativas à posição do robô.

O EKF provou-se uma técnica eficiente na estimação da localização do robô, apresentado melhor resultado em relação ao HMM. Isto se deve em grande parte ao fato do HMM não ter fase de predição, e só realizar a estimação da posição quando uma nova medida é recebida. Enquanto, o EKF pondera entre a predição e a medição para convergir para a trajetória real executada pelo robô.

Nos testes, a taxa de atualização da medição foi de $500 \mathrm{~ms}$, se esta taxa for diminuída o resultado dos estimadores melhora, devido ao aumento de informações. Os experimentos com a trajetória do "oito" e o "caracol de Pascal" foram mais consistentes, pois apresentam curvas suaves. Concomitante, a trajetória do "quadrado" apresentou avarias por possuir uma curva de $90^{\circ}$. Neste trabalho a medição é constituída do par $(x, y)$, e não realiza-se a medição do ângulo do robô, por isto ocorreram grandes alterações nas curvas acentuadas.
Há de se salientar que na replicação da aplicação desenvolvida para um ambiente real deve-se atentar para alguns parâmetros dos transceptores que não foram levados em conta na simulação. Por exemplo, na comunicação dos transceptores há atrasos no tempo de resposta, que podem ter várias fontes, como atrasos na aquisição e propagação do sinal, e também atrasos no software de processamento. Todos estes dados devem ser analisados para se validar a precisão das medidas obtidas com um hardware real.

Como trabalhos futuros pretende-se verificar a robustez dos estimadores HMM e EKF quando um dos transceptores está em estado de falha, ou operando de forma intermitente. Outra melhoria seria incluir a informação da posição angular do robô na medição.

\section{REFERÊNCIAS}

Cassandras, C.G. and Lafortune, S. (2009). Introduction to discrete event systems. Springer Science \& Business Media.

Chong, K.S. and Kleeman, L. (1997). Accurate odometry and error modelling for a mobile robot. In Robotics and Automation, 1997. Proceedings., 1997 IEEE International Conference on, volume 4, 2783-2788. IEEE.

Forney, G.D. (1973). The viterbi algorithm. Proceedings of the IEEE, 61(3), 268-278.

Lee, S.H., Lee, H.C., and Lee, B.H. (2011). Visuallyextended vector polar histogram applied to robot route navigation. International Journal of Control, Automation and Systems, 9(4), 726.

Norrdine, A. (2012). An algebraic solution to the multilateration problem. In Proceedings of the 15th international conference on indoor positioning and indoor navigation, Sydney, Australia, volume 1315.

Peter, O. (2016). Learning Complex Markov Models for Mobile Robotics. Ph.D. thesis, PhD thesis, University of Oxford, England.

Pomárico-Franquiz, J., Khan, S.H., and Shmaliy, Y.S. (2014). Combined extended fir/kalman filtering for indoor robot localization via triangulation. Measurement, 50, 236-243.

Rabiner, L.R. (1990). A tutorial on hidden markov models and selected applications in speech recognition. In Readings in speech recognition, 267-296. Elsevier.

Särkkä, S. (2013). Bayesian filtering and smoothing, volume 3. Cambridge University Press.

Savage, J., Fuentes, O., Contreras, L., and Negrete, M. (2018). Map representation using hidden markov models for mobile robot localization. In MATEC Web of Conferences, volume 161, 03011. EDP Sciences.

Spong, M.W., Hutchinson, S., Vidyasagar, M., et al. (2006). Robot modeling and control, volume 3. Wiley New York.

Thrun, S., Burgard, W., and Fox, D. (2005). Probabilistic robotics. MIT press.

Tong, X., Li, Z., Han, G., Liu, N., Su, Y., Ning, J., and Yang, F. (2018). Adaptive ekf based on hmm recognizer for attitude estimation using mems marg sensors. IEEE Sensors Journal, 18(8), 3299-3310.

Viterbi, A. (1967). Error bounds for convolutional codes and an asymptotically optimum decoding algorithm. IEEE transactions on Information Theory, 13(2), 260269. 